\title{
In search of goldilocks: the quest to optimize combination drug strategies for the management of advanced stage non-small-cell lung cancer
}

\author{
Poorva Bindal, Page Widick, Daniel B. Costa, Deepa Rangachari \\ Department of Medicine, Division of Medical Oncology, Beth Israel Deaconess Medical Center, Harvard Medical School, Boston, MA, USA \\ Correspondence to: Deepa Rangachari, MD. Division of Medical Oncology, Beth Israel Deaconess Medical Center, 330 Brookline Avenue, Boston, MA \\ 02215, USA. Email: drangach@bidmc.harvard.edu. \\ Comment on: West H, McCleod M, Hussein M, et al. Atezolizumab in combination with carboplatin plus nab-paclitaxel chemotherapy compared with \\ chemotherapy alone as first-line treatment for metastatic non-squamous non-small-cell lung cancer (IMpower130): a multicenter, randomized, open- \\ label, phase 3 trial. Lancet Oncol 2019;20:924-37.
}

Submitted Dec 20, 2019. Accepted for publication Jan 21, 2020.

doi: $10.21037 /$ tcr.2020.01.20

View this article at: http://dx.doi.org/10.21037/tcr.2020.01.20

\section{Introduction}

The past two decades have seen significant therapeutic advances in the management of advanced non-small cell lung cancer (NSCLC): from histology-focused chemotherapy regimens to personalized medicine where each patient is treated in accordance with the unique genetic and molecular features of their tumor. The evidence-based standard of care for frontline palliative systemic therapy in advanced NSCLC includes: (I) tyrosine kinase inhibitors (TKIs) for those with actionable alterations in epidermal growth factor receptor (EGFR), anaplastic lymphoma kinase (ALK), ROS proto-oncogene 1 (ROS1), or B-Raf proto-oncogene (BRAF); or (II) immune checkpoint inhibitors (ICIs) alone or in combination with platinum doublet chemotherapy for those without actionable genetic alterations (1). Current expert guidelines recommend testing for alterations in EGFR, ALK, ROS1, and BRAF at a minimum in advanced stage lung adenocarcinomas and tumor programmed death ligand-1 (PD-L1) immunohistochemistry for all advanced NSCLCs (2).

However, for the significant proportion $(\sim 5 \%)$ of patients with advanced NSCLC whose tumors do not harbor sensitizing genetic alterations, identifying more efficacious and tolerable strategies beyond sequential use of cytotoxic chemotherapies was an area of significant unmet need. Since the approval of ICIs by the United States Food and Drug Administration (FDA) in 2015, numerous ongoing efforts have focused on evolving better strategies for this large cohort of patients by incorporating immunebased therapies-either alone or in combination with other chemotherapeutic, immunologic, and/or biologic agents (3). In this editorial, we explore the recent explosion of data regarding immune-based combination therapies, placing the recently published IMpower 130 trial by West et al. into context, and emphasizing strategies to optimize their use.

\section{The quest for a predictive biomarker: PD-L1 and beyond}

For patients whose tumors lack actionable driver oncogene alterations, PD-L1 tumor proportion score (TPS) remains the best-vetted biomarker for therapeutic selection. Even so, its limitations are many, and use of a singular biomarker in this regard is likely an oversimplification of the tumorimmune axis. Specifically, durable responses with ICIs have been seen in patients with low/absent tumor PD-L1 expression, and objective response rates (ORRs) even in those with high (i.e., TPS $\geq 50 \%$ ) PD-L1 is on the order of $50 \%$ $(4,5)$. There is also growing awareness regarding the differing operating characteristics of the various PD-L1 assays and temporospatial heterogeneity in PD-L1 expression $(6,7)$.

Tumor mutation burden (TMB) has also drawn recent interest, particularly given the association with tobacco as 
a potent carcinogen and recognition that lung cancers rank amongst the tumors with the highest burden of somatic mutations (8). Increasing numbers of somatic mutations lead to the production of unique tumor neoantigens that can be leveraged by the native immune system to recognize and kill malignant cells $(3,9)$. Several studies have shown that tumors expressing high PD-L1 or TMB may represent mutually exclusive populations-i.e., one is not synonymous with the other (10). However, despite its biologic plausibility, the utility of TMB as a biomarker has been hampered by several recent exploratory analyses showing no statistically significant association between TMB and outcomes of ICI with/without chemotherapy $(11,12)$. Additional uncertainties regarding TMB use include defining TMB thresholds and methods. As noted previously, there is growing recognition of intratumoral heterogeneity such that validation of peripheral bloodbased assays may be more informative than tissue-based testing. A recent retrospective analysis of two randomized trials showed that blood-based TMB might be a promising alternate predictive biomarker (13).

TMB and PD-L1 aside, the field is necessarily moving towards a more nuanced understanding of determining optimal first line therapeutic options in the significant proportion of patients without actionable genetic alterations and with low/absent tumor PD-L1. Assessment of tumor microsatellite instability, mutations in DNA damage repair pathways, tumor-infiltrating lymphocytes, tobacco exposure, tumor co-mutation profile, and other genetic determinants of host immunity all remain areas of intensive investigation, and a multifaceted approach to therapeutic stratification in these patients will likely be a feasible imperative in the future (14).

\section{Identifying patients in need of combination therapy strategies: when is more really better?}

Given their potential for durable and tolerable benefits, there has been considerable interest in use of single agent ICI regimens for those whose tumors harbor no actionable genetic alterations. KEYNOTE-024 established single agent pembrolizumab as a standard of care in those with TPS $\geq 50 \%(5,15)$. Subsequently, KEYNOTE-042 showed an OS benefit with pembrolizumab monotherapy in the overall study population (TPS $\geq 1 \%$ ), though this benefit was primarily driven by patients with PD-L1 TPS $\geq 50 \%$; an exploratory analysis in patients with TPS $1-49 \%$ did not demonstrate a significant OS advantage (16). This data notwithstanding, the FDA has approved single agent pembrolizumab for any advanced NSCLC with TPS $\geq 1 \%$. The ongoing IMpower 110 [NCT02409342] and MYSTIC [NCT02453282] trials are similarly evaluating other upfront single agent ICI strategies.

However, a significant proportion of patients-nearly $50 \%$ - with advanced NSCLC will not derive benefit from single agent regimens. In numerous contemporaneous studies [KEYNOTE-189, KEYNOTE-407, IMpower 130, IMpower 131, IMpower 132, IMpower 150 (17-22)], combining ICIs with a platinum doublet has demonstrated notable improvements in OS across all subgroups of patients (irrespective of tumor PD-L1 status) - though this comes not without cost as it relates to clinical and financial toxicities.

Thus, an important question emerges: When is more really better, and for whom are combination strategies absolutely needed?

\section{Combination therapy strategies: an increasingly crowded landscape}

\section{IMpower 130 and other ICI + chemotherapy combinations}

Combination of ICIs with cytotoxic chemotherapeutic agents is a biologically attractive approach, given the potential to augment antigen presentation and reduce immunosuppressive activity in the tumor microenvironment $(23,24)$. Consequently, rapidly emerging clinical trial data has led to approval of several ICI combinations for first line treatment of advanced NSCLC. It is into this context that West et al. deliver IMpower 130. In this phase III, internationally-conducted trial, 724 patients were randomized in a $2: 1$ fashion to receive combination carboplatin/nab-paclitaxel with atezolizumab every 3 weeks for up to 6 cycles followed by atezolizumab maintenance $v s$. carboplatin/nab-paclitaxel followed by best supportive care (19). Patients with EGFR/ALK+ disease were permitted if they had disease progression on/intolerance to prior TKI therapies, as were those with treated asymptomatic brain metastases. With a median follow up that exceeds 18 months, median PFS and OS were both improved with a median OS of 18.6 vs. 13.9 months (HR 0.79, 95\% CI: $0.64-0.98, \mathrm{P}=0.033$ ) with chemoimmunotherapy $v s$. chemotherapy alone, respectively; the ORR was also increased from $31.9 \%$ to $49.2 \%$ with combination chemoimmunotherapy and with improved median duration of response (DoR) with the combination (8.4 vs. 6.1 months). Survival benefit was sustained across all analyzed subgroups-with the exception of those with liver metastases and EGFR/ALK alterations. Hazard ratios for 
OS were comparable across all PD-L1 subgroups. Grade 3-4 adverse events (AEs) were seen in $81 \%$ of patients treated with chemoimmunotherapy $v s .71 \%$ of patients treated with chemotherapy alone and with similar rates of AE-related treatment discontinuation. Immune-related AEs were specifically seen in $45 \%$ of patients treated with combination chemoimmunotherapy, mostly grade $1-2$. No new safety signals were identified.

Similar to IMpower 130, multiple other phase III studies have shown favorable trends in survival outcomes with tolerable safety profile for frontline ICI with platinum doublet in all patients with advanced NSCLC-regardless of tumor PD-L1 status-in non-squamous (KEYNOTE-189, IMpower 132) and squamous (KEYNOTE-407, IMpower 131) disease alike $(17,18,20,21,25)$. On the basis of these iterative studies, pembrolizumab and atezolizumab have both garnered FDA approval for use in combination with platinum doublet chemotherapy for frontline management of advanced NSCLC in those lacking actionable alterations.

Notably, in each of these ICI only or ICI + chemotherapy studies, the comparator arm has been the now defunct historical standard of platinum doublet chemotherapy alone. Hence, it remains uncertain: Is combination chemoimmunotherapy optimal for all patients without sensitizing genetic alterations? Are upfront combination strategies superior to sequential therapies?

\section{ICI + biologic combinations}

Bevacizumab has existed within the armamentarium for advanced NSCLC for use in combination with platinum doublet chemotherapy and/or subsequent maintenance therapy for more than a decade. In a randomized phase II study, addition of bevacizumab to carboplatin/paclitaxel followed by bevacizumab maintenance in patients with advanced/recurrent NSCLC led to increased ORR (31.5\% vs. $18.8 \%)$ and prolonged time to progression (7.4 vs. 4.2 months; $\mathrm{P}=0.023)$ compared to chemotherapy alone. A higher incidence of bleeding was noted in the bevacizumab group, and severe pulmonary hemorrhage occurred in 6 patients (including 4 fatalities); this led to the restriction of bevacizumab's use to non-squamous tumors only (26). A subsequent phase III trial (E4599) confirmed the PFS (6.2 vs. 4.5 months, HR 0.66, $\mathrm{P}<0.001)$ and $\mathrm{OS}(12.3$ vs. 10.3 months, HR $0.79, \mathrm{P}=0.003)$ benefit with bevacizumabcontaining regimens and led to bevacizumab's FDA approval (27). Even so, use of bevacizumab in routine clinical practice for patients with advanced NSCLC has been significantly limited by its toxicity profile-particularly given the context of an older, comorbid population in whom this modest survival benefit does not afford a favorable therapeutic window.

In the immunotherapy era, bevacizumab and like agents are again the subjects of investigation given their known immunomodulatory effects. Vascular endothelial growth factor (VEGF) influences immune cells in the tumor microenvironment by promoting immunosuppressive cells (regulatory T cells, myeloid-derived suppressor cells) and inhibiting antigen-presenting cells (dendritic cells), thereby rendering the tumor microenvironment immunosuppressed. Thus, VEGF inhibitors are attractive as a means to potentiate ICI effects by reversing immunosuppressive mechanisms contributing to immune escape and proliferation (28).

In the IMpower150 trial, patients with recurrent/advanced non-squamous NSCLC were randomized to three subgroups: atezolizumab/bevacizumab/carboplatin/paclitaxel (ABCP), atezolizumab/carboplatin/paclitaxel (ACP), and bevacizumab/ carboplatin/paclitaxel (BCP)—regardless of tumor PD-L1 status. EGFR/ALK+ patients with TKI-refractory disease were eligible (but were excluded from the primary endpoint analyses). To date, outcomes for the ABCP and BCP groups have been presented and with significant improvement in investigator-assessed PFS (median 8.3 vs. 6.8 months; HR 0.62; 95\% CI: $0.52-0.74 ; \mathrm{P}<0.001$ ) and OS (median OS 19.2 vs. 14.7 months; HR 0.78; 95\% CI: 0.64-0.96; $\mathrm{P}=0.02$ ) with $\mathrm{ABCP} v s$. BCP, respectively. This benefit was maintained across patients with all levels of tumor PD-L1 expression (22). Notably, in an expanded subgroup analysis, the chemoimmunotherapy + bevacizumab combination has been the first to show improved OS in patients with EGFR/ALK+ disease (29). However, no OS benefit was seen with ACP vs. BCP in this subgroup, suggesting that there may be a specific role for VEGF-mediated immune modulation in this specific subset. Given the limited numbers, additional prospective evaluation is needed. On the basis of this study, bevacizumab is now FDA approved in combination with carboplatin/ paclitaxel/atezolizumab. However, as the outcomes in the overall population for chemoimmunotherapy + bevacizumab $v s$. chemoimmunotherapy alone have not yet been published, a recurrent theme emerges: Which groups of patients derive optimal benefit from these intensified combination strategies?

\section{ICI + ICI combinations}

Given the success of ICIs in terms of efficacy, toxicity, and quality of life, there has been increasing interest amongst 
patients and providers alike to identify chemotherapysparing regimens. As both PD-1 and CTLA-4 modulate T-cell function through distinct-but complementarypathways, combination ICI + ICI strategies are also under investigation.

CHECKMATE-227 is a multi-arm, phase III, randomized controlled trial exploring several nivolumab-containing strategies $v s$. platinum doublet chemotherapy in patients with advanced/recurrent NSCLC and stratified on the basis of tumor PD-L1 expression. In those with absent PD-L1 (TPS $<1 \%$ ), patients were randomized 1:1:1 to: (I) nivolumab + chemotherapy, (II) nivolumab + ipilimumab, or (III) chemotherapy alone. In those with tumor PD-L1 TPS $\geq 1 \%$, patients were randomized 1:1:1 to: (I) nivolumab alone, (II) nivolumab + ipilimumab, or (III) chemotherapy alone. Improved OS was seen in those receiving nivolumab + ipilimumab $v s$. chemotherapy alone, regardless of tumor PD-L1 status (PD-L1 TPS <1\%: median OS 17.2 vs. 12.2 months, HR 0.62, 95\% CI: 0.48-0.78; PD-L1 TPS $\geq 1 \%$ : 17.1 vs. 14.9 months, HR $0.79,97.72 \%$ CI: 0.65-0.96) (30). As has been seen in other trials, when responses occur, they tend to be far more durable with ICIs than with chemotherapy: median DoR with nivolumab + ipilimumab vs. chemotherapy was 23.2 vs. 6.2 months (PD-L1 TPS $\geq 1 \%$ ) and 18.0 vs. 4.8 months (PD-L1 TPS $<1 \%$ ), respectively (30). The ongoing NEPTUNE trial (NCT02542293) is exploring the efficacy and safety of another ICI combination (durvalumab + tremelimumab) as first line therapy.

Notably, in these as in all of the other studies outlined above, the comparator arm has been platinum doublet chemotherapy alone. Given the now well-established benefits of ICIs as part of the upfront therapeutic strategy for all eligible patients with advanced NSCLC lacking actionable genomic alterations, the relative benefits of ICI + ICI $v s$. ICI + chemotherapy combinations remain uncertain, and additional questions emerge: Are there cases where chemotherapy can be reasonably omitted from combination therapy strategies?

\section{Clinical and financial toxicities: an occupational hazard of combination strategies}

In an era when more tolerable and efficacious therapies may translate into a longer duration of therapy, it has become increasingly imperative to carefully weigh improvements in clinical outcomes against tradeoffs with regard to both clinical and financial toxicities-and in doing so, to ensure optimized, sustainable care to all who may benefit (Table 1).

A meta-analysis of 14 recent clinical trials demonstrated the superior efficacy of combining ICIs with chemotherapy compared with chemotherapy alone in terms of tumor response and long-term survival (32). However, the pooled results also revealed significant increases in clinical toxicities as compared with the administration of chemotherapy alone[relative risk (RR) 1.11; 95\% CI: 1.04-1.18] (32). More patients receiving chemoimmunotherapy discontinued their treatment due to toxicity as compared to those receiving chemotherapy alone (RR 1.46; 95\% CI: 1.23-1.74) (32). Grade 3 or higher drug-related AEs have been reported in detail in the various combination therapy studies to date: $60-80 \%$ for chemoimmunotherapy regimens $(17,19-21,25)$, $58.5 \%$ for bevacizumab + chemoimmunotherapy (22), and $31.2 \%$ for ICI + ICI regimens (31). Despite the higher rates of moderate-severe treatment-related AEs seen in these studies, however, health-related quality of life (QoL) and patient-reported outcomes (PROs) do not appear to have been compromised in several of these trials (33-36).

Cost effectiveness analyses additionally serve as important tools to assess whether novel therapeutic strategies provide clinical benefit at a justifiable cost. In such an analysis of first line ICI, pembrolizumab was found to be cost effective at a willingness-to-pay threshold of $\$ 180,000$ /quality adjusted life year (QALY) with an incremental cost effectiveness ratio (ICER) of US $\$ 104,823 /$ QALY when compared to chemotherapy (37). First line pembrolizumab monotherapy was also projected to be cost effective compared to platinum doublet chemotherapy for patients with PD-L1 TPS $\geq 1 \%$, with an expected gain of 0.60 QALY and ICER US \$130,155/ QALY (38). In a simulated economic analysis, treatment with $\mathrm{ABCP}$ was compared with $\mathrm{BCP}$, and addition of atezolizumab to BCP was associated with a mean survival of 2.13 QALYs/patient for an estimated ICER of \$201,676/QALY. Pembrolizumab combination therapy was also compared to platinum doublet therapy alone and was noted to have greater incremental QALYs at a lower incremental cost (mean survival 2.45 QALY/patient for ICER \$116,698/QALY) (39). Thus, despite clinical benefits seen with ICI combination strategies, further price reductions may be needed before they can be universally adopted in all healthcare settings.

\section{Conclusion: in search of Goldilocks and the path toward optimal care}

After many decades, the hegemony of the platinum doublet 


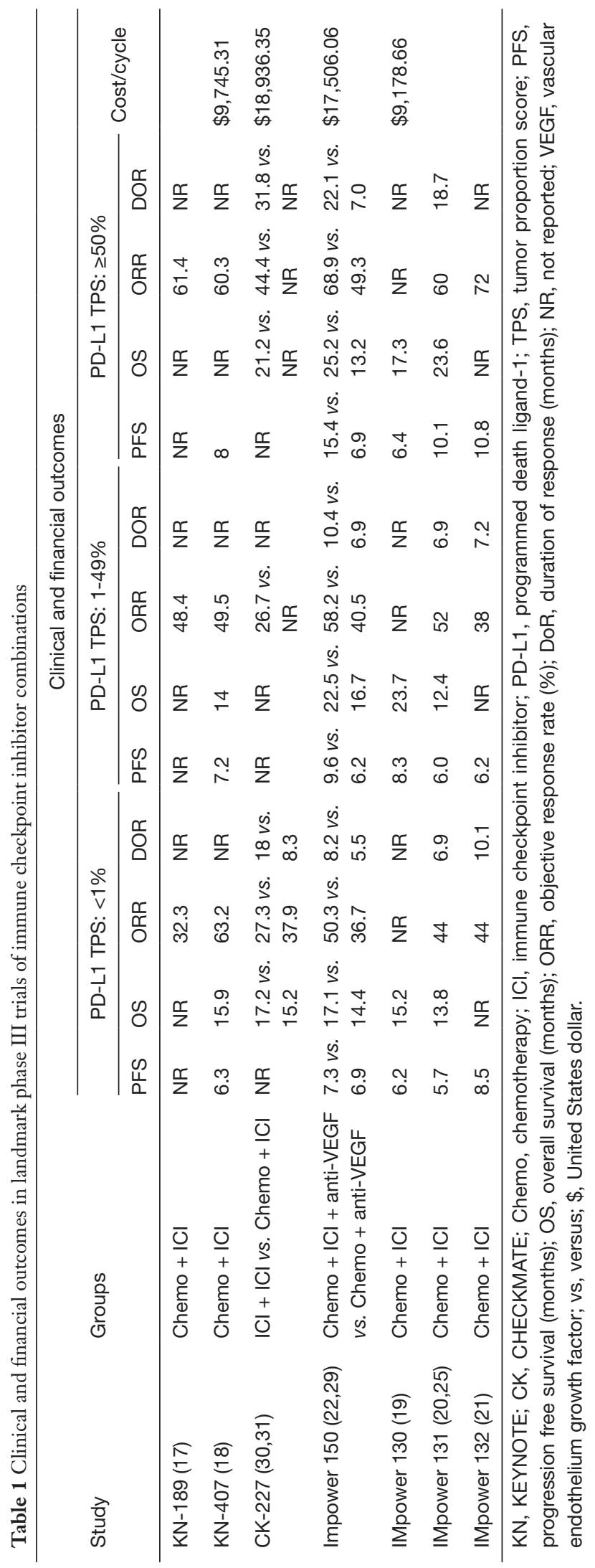

has been surpassed, and a new era defined by the use of targeted and immune-based therapies has dramatically altered the therapeutic landscape for patients with advanced NSCLC. For the large proportion of patients whose tumors lack an actionable genomic alteration, an unmet need has finally found hope, and a growing proportion of patients have been afforded durably efficacious and tolerable therapies with incorporation of ICIs-either alone or in combination with other agents.

Despite rapidly emerging clinical trial data and a steady stream of drug approvals, it will take considerable ongoing effort to rationally stratify patients to care regimens that achieve equipoise with regard to the combinations/intensity of therapies used and optimal clinical outcomes. With such progress necessarily comes a need for heightened expectations with regard to managing the financial and societal impacts of care so that all who may benefit are afforded the opportunity for best care. In our own practice, we continue to favor stratifying frontline therapy for treatment-eligible patients with advanced stage NSCLC in the following manner: (I) if actionable genomic alteration, then TKI; (II) if tumor PD-L1 TPS $\geq 50 \%$, then single agent pembrolizumab; or (III) if tumor PD-L1 TPS $<50 \%$, then combination chemoimmunotherapy. Pending a better understanding of who may most benefit from or require such approaches, we most often defer use of other approved/ evolving combinations (i.e., chemoimmunotherapy + bevacizumab and ICI + ICI) (Figure 1). Whether sequential use of these agents and/or in combination with other immune-augmenting strategies might further amplify benefit in specific subgroups and with manageable toxicities remains to be seen and is the subject of numerous ongoing trials: INSIGNIA (NCT03793179, concurrent vs. sequential pembrolizumab + platinum doublet), CheckMate-9LA (NCT03215706, nivolumab + ipilimumab + platinum doublet), and NCT02407171 (pembrolizumab + stereotactic body radiotherapy) amongst others.

As a community, we must work towards continued refinement of our therapeutic paradigm, with evolution of a multiplex model for patient-centered care that incorporates histology, genomic and molecular profiles, clinical characteristics, toxicities, economic/logistical factors, and other patient-specific criteria in the most optimal way. Without doing so, the considerable progress that has been made will fall short of its full potential. Like Goldilocks, we, too, must get it "just right". 


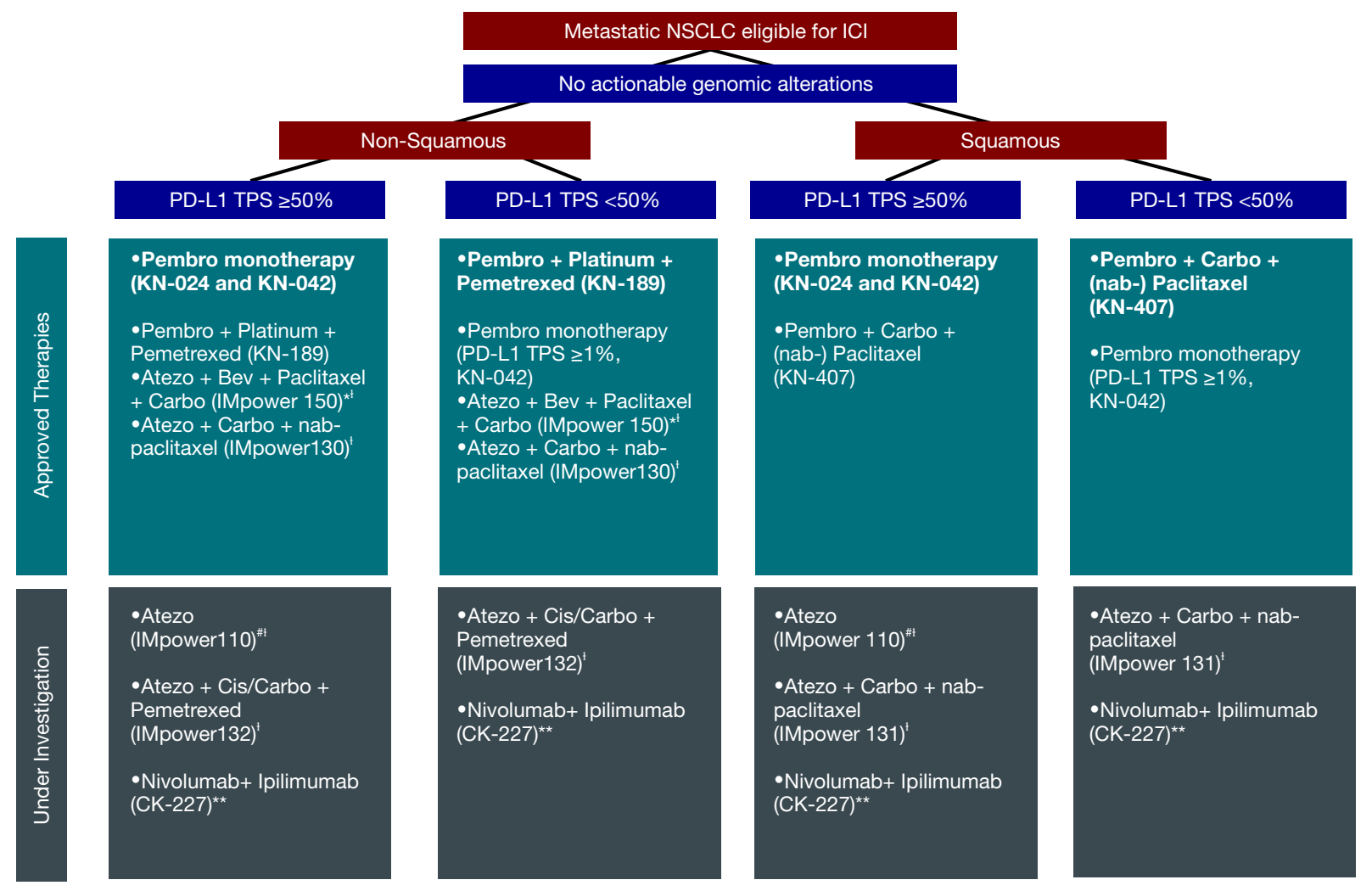

Figure 1 Therapeutic stratification for frontline therapy in advanced non-small-cell lung cancer: current and evolving standards. NSCLC, non-small-cell lung cancer; PD-L1, programmed death ligand-1; TPS, tumor proportion score; Pembro, Pembrolizumab; Atezo, Atezolizumab; Bev, Bevacizumab; Carbo, Carboplatin; Cis, Cisplatin; KN, KEYNOTE. Bolded print denotes authors' approach. *Benefit also seen in patients with liver metastases and TKI-refractory EGFR/ALK+ disease. 'PD-L1 evaluated using VENTANA SP142 assay, with staining of tumor cells + tumor-infiltrating immune cells. ${ }^{* *}$ Progression-free survival benefit transcended PD-L1 expression and was equal in PD-L1 $\geq$ or $<1 \%$. "IMpower110: NCT02409342.

\section{Acknowledgments}

Funding: None.

\section{Footnote}

Provenance and Peer Review: This article was commissioned by the editorial office, Translational Cancer Research. The article did not undergo external peer review.

Conflicts of Interest: Dr. Rangachari reports nonfinancial support (institutional research support) from BristolMyers Squibb, Novocure, and Abbvie/Stemcentrx, all outside the submitted work. Dr. Costa reports personal fees (consulting fees and honoraria) and nonfinancial support (institutional research support) from Takeda/Millennium Pharmaceuticals, and AstraZeneca, and Pfizer, as well as nonfinancial support (institutional research support) from Merck Sharp and Dohme Corporation, Merrimack Pharmaceuticals, Bristol-Myers Squibb, Clovis Oncology, and Tesaro, all outside the submitted work. Drs. Bindal and Widick have no conflicts of interest to declare.

Ethical Statement: The authors are accountable for all aspects of the work in ensuring that questions related to the accuracy or integrity of any part of the work are appropriately investigated and resolved.

Open Access Statement: This is an Open Access article distributed in accordance with the Creative Commons Attribution-NonCommercial-NoDerivs 4.0 International License (CC BY-NC-ND 4.0), which permits the noncommercial replication and distribution of the article with 
the strict proviso that no changes or edits are made and the original work is properly cited (including links to both the formal publication through the relevant DOI and the license). See: https://creativecommons.org/licenses/by-nc-nd/4.0/.

\section{References}

1. Herbst RS, Morgensztern D, Boshoff C. The biology and management of non-small cell lung cancer. Nature 2018;553:446-54.

2. Lindeman NI, Cagle PT, Aisner DL, et al. Updated Molecular Testing Guideline for the Selection of Lung Cancer Patients for Treatment With Targeted Tyrosine Kinase Inhibitors: Guideline From the College of American Pathologists, the International Association for the Study of Lung Cancer, and the Association for Molecular Pathology. J Thorac Oncol 2018;13:323-58.

3. Doroshow DB, Sanmamed MF, Hastings K, et al. Immunotherapy in Non-Small Cell Lung Cancer: Facts and Hopes. Clin Cancer Res 2019;25:4592-602.

4. Garon EB, Hellmann MD, Rizvi NA, et al. Five-Year Overall Survival for Patients With Advanced Non-SmallCell Lung Cancer Treated With Pembrolizumab: Results From the Phase I KEYNOTE-001 Study. J Clin Oncol 2019;37:2518-27.

5. Reck M, Rodriguez-Abreu D, Robinson AG, et al. Pembrolizumab versus Chemotherapy for PD-L1Positive Non-Small-Cell Lung Cancer. N Engl J Med 2016;375:1823-33

6. Hirsch FR, McElhinny A, Stanforth D, et al. PDL1 Immunohistochemistry Assays for Lung Cancer: Results from Phase 1 of the Blueprint PD-L1 IHC Assay Comparison Project. J Thorac Oncol 2017;12:208-22.

7. Hong L, Dibaj S, Negrao MV, et al. Spatial and temporal heterogeneity of PD-L1 and its impact on benefit from immune checkpoint blockade in non-small cell lung cancer (NSCLC). J Clin Oncol 2019;37:9017.

8. Alexandrov LB, Nik-Zainal S, Wedge DC, et al. Signatures of mutational processes in human cancer. Nature 2013;500:415-21.

9. Schumacher TN, Scheper W, Kvistborg P. Cancer Neoantigens. Annu Rev Immunol 2019;37:173-200.

10. Rizvi H, Sanchez-Vega F, La K, et al. Molecular Determinants of Response to Anti-Programmed Cell Death (PD)-1 and Anti-Programmed Death-Ligand 1 (PD-L1) Blockade in Patients With Non-Small-Cell Lung Cancer Profiled With Targeted Next-Generation Sequencing. J Clin Oncol 2018;36:633-41.
11. Herbst R, Lopes G, Kowalski D, et al. LBA79 Association between tissue TMB (tTMB) and clinical outcomes with pembrolizumab monotherapy (pembro) in PD-L1-positive advanced NSCLC in the KEYNOTE-010 and-042 trials. Ann Oncol 2019;30:mdz394.077.

12. Paz-Ares L, Langer C, Novello S, et al. LBA80 Pembrolizumab (pembro) plus platinum-based chemotherapy (chemo) for metastatic NSCLC: Tissue TMB (tTMB) and outcomes in KEYNOTE-021, 189, and 407. Ann Oncol 2019;30:mdz394.078.

13. Gandara DR, Paul SM, Kowanetz M, et al. Bloodbased tumor mutational burden as a predictor of clinical benefit in non-small-cell lung cancer patients treated with atezolizumab. Nat Med 2018;24:1441-8.

14. Havel JJ, Chowell D, Chan TA. The evolving landscape of biomarkers for checkpoint inhibitor immunotherapy. Nat Rev Cancer 2019;19:133-50.

15. Reck M, Rodriguez-Abreu D, Robinson AG, et al. Updated Analysis of KEYNOTE-024: Pembrolizumab Versus Platinum-Based Chemotherapy for Advanced NonSmall-Cell Lung Cancer With PD-L1 Tumor Proportion Score of 50\% or Greater. J Clin Oncol 2019;37:537-46.

16. Mok TSK, Wu YL, Kudaba I, et al. Pembrolizumab versus chemotherapy for previously untreated, PD-L1expressing, locally advanced or metastatic non-small-cell lung cancer (KEYNOTE-042): a randomised, open-label, controlled, phase 3 trial. Lancet 2019;393:1819-30.

17. Gandhi L, Rodriguez-Abreu D, Gadgeel S, et al. Pembrolizumab plus Chemotherapy in Metastatic NonSmall-Cell Lung Cancer. N Engl J Med 2018;378:2078-92.

18. Paz-Ares L, Luft A, Vicente D, et al. Pembrolizumab plus Chemotherapy for Squamous Non-Small-Cell Lung Cancer. N Engl J Med 2018;379:2040-51.

19. West H, McCleod M, Hussein M, et al. Atezolizumab in combination with carboplatin plus nab-paclitaxel chemotherapy compared with chemotherapy alone as firstline treatment for metastatic non-squamous non-smallcell lung cancer (IMpower130): a multicentre, randomised, open-label, phase 3 trial. Lancet Oncol 2019;20:924-37.

20. Jotte RM, Cappuzzo F, Vynnychenko I, et al. IMpower131: Primary PFS and safety analysis of a randomized phase III study of atezolizumab+ carboplatin+ paclitaxel or nab-paclitaxel vs carboplatin+ nab-paclitaxel as $1 \mathrm{~L}$ therapy in advanced squamous NSCLC. J Clin Oncol 2018;36:LBA9000.

21. Papadimitrakopoulou V, Cobo M, Bordoni R, et al. IMpower132: PFS and safety results with $1 \mathrm{~L}$ atezolizumab+ carboplatin/cisplatin+ pemetrexed in stage 
IV non-squamous NSCLC. J Thorac Oncol 2018;13.

22. Socinski MA, Jotte RM, Cappuzzo F, et al. Atezolizumab for First-Line Treatment of Metastatic Nonsquamous NSCLC. N Engl J Med 2018;378:2288-301.

23. Herbst RS, Sznol M. Diminished but not dead: chemotherapy for the treatment of NSCLC. Lancet Oncol 2016;17:1464-5.

24. Melosky B, Juergens R, Hirsh V, et al. Amplifying Outcomes: Checkpoint Inhibitor Combinations in First-Line Non-Small Cell Lung Cancer. Oncologist 2020;25:64-77.

25. Jotte R, Cappuzzo F, Vynnychenko I, et al. OA14. 02 IMpower131: Final OS Results of Carboplatin+ NabPaclitaxel \pm Atezolizumab in Advanced Squamous NSCLC. J Thorac Oncol 2019;14:S243-4.

26. Johnson DH, Fehrenbacher L, Novotny WF, et al. Randomized phase II trial comparing bevacizumab plus carboplatin and paclitaxel with carboplatin and paclitaxel alone in previously untreated locally advanced or metastatic non-small-cell lung cancer. J Clin Oncol 2004;22:2184-91.

27. Sandler A, Gray R, Perry MC, et al. Paclitaxel-carboplatin alone or with bevacizumab for non-small-cell lung cancer. N Engl J Med 2006;355:2542-50.

28. Jain RK. Antiangiogenesis strategies revisited: from starving tumors to alleviating hypoxia. Cancer cell 2014;26:605-22.

29. Reck M, Mok TSK, Nishio M, et al. Atezolizumab plus bevacizumab and chemotherapy in non-small-cell lung cancer (IMpower150): key subgroup analyses of patients with EGFR mutations or baseline liver metastases in a randomised, open-label phase 3 trial. Lancet Respir Med 2019;7:387-401.

30. Hellmann MD, Paz-Ares L, Bernabe Caro R, et al. Nivolumab plus Ipilimumab in Advanced Non-Small-Cell Lung Cancer. N Engl J Med 2019;381:2020-31.

31. Hellmann MD, Ciuleanu TE, Pluzanski A, et al. Nivolumab plus Ipilimumab in Lung Cancer with a High Tumor Mutational Burden. N Engl J Med 2018;378:2093-104.

Cite this article as: Bindal P, Widick P, Costa DB, Rangachari D. In search of goldilocks: the quest to optimize combination drug strategies for the management of advanced stage non-smallcell lung cancer. Transl Cancer Res 2020;9(3):1311-1318. doi: 10.21037/tcr.2020.01.20
32. Chen Y, Zhou Y, Tang L, et al. Immune-Checkpoint Inhibitors as the First Line Treatment of Advanced NonSmall Cell Lung Cancer: A Meta-Analysis of Randomized Controlled Trials. J Cancer 2019;10:6261-8.

33. Garassino MC, Rodriguez-Abreu D, Gadgeel SM, et al. Health-related quality of life (HRQoL) in the KEYNOTE-189 study of pembrolizumab (pembro) or placebo (pbo) + pemetrexed (pem) + platinum (plt) for metastatic NSCLC. J Clin Oncol 2018;36:9021.

34. Mazieres J, Kowalski D, Luft A, et al. Health-Related Quality of Life With Carboplatin-Paclitaxel or nabPaclitaxel With or Without Pembrolizumab in Patients With Metastatic Squamous Non-Small-Cell Lung Cancer. J Clin Oncol 2020;38:271-80.

35. Reck M, Karagiannis T, Wehler T, et al. Patientreported outcomes (PROs) in the randomized, phase III IMpower150 study of atezolizumab (atezo) + chemotherapy (chemo) \pm bevacizumab (bev) vs chemo + bev in 1L nonsquamous metastatic NSCLC (mNSCLC). J Clin Oncol 2018;36:9047.

36. Reck M, Schenker M, Lee KH, et al. Nivolumab plus ipilimumab versus chemotherapy as first-line treatment in advanced non\&\#x2013;small-cell lung cancer with high tumour mutational burden: patient-reported outcomes results from the randomised, open-label, phase III CheckMate 227 trial. Eur J Cancer 2019;116:137-47.

37. Insinga RP, Vanness DJ, Feliciano JL, et al. Costeffectiveness of pembrolizumab in combination with chemotherapy in the 1st line treatment of non-squamous NSCLC in the US. J Med Econ 2018;21:1191-205.

38. Huang M, Lopes GL, Insinga RP, et al. Cost-effectiveness of pembrolizumab versus chemotherapy as first-line treatment in PD-L1-positive advanced non-small-cell lung cancer in USA. Immunotherapy 2019;11:1463-78.

39. Criss SD, Mooradian MJ, Watson TR, et al. Costeffectiveness of Atezolizumab Combination Therapy for First-Line Treatment of Metastatic Nonsquamous NonSmall Cell Lung Cancer in the United States. JAMA Netw Open 2019;2:e1911952. 